gr-qc/9506054

$\mathrm{DTP} / 95 / 37$

SUSX-TH- $95 / 73$

\title{
SMOOTH METRICS FOR SNAPPING STRINGS
}

\author{
Ruth Gregory \\ Department of Applied Mathematics and Theoretical Physics, \\ University of Cambridge, Silver St, Cambridge, CB3 9EW, U.K. \\ $\&$ \\ Centre for Particle Theory, \\ Durham University, South Road, Durham, DH1 3LE, U.K. \\ and \\ Mark Hindmarsh \\ School of Mathematical and Physical Sciences, \\ University of Sussex, Brighton BN1 9QH,UK
}

\begin{abstract}
We construct two possible metrics for abelian Higgs vortices with ends on black holes. We show how the detail of the vortex fields smooths out the nodal singularities which exist in the idealized metrics. A corollary is that apparently topologically stable strings might be able to split by black hole pair production. We estimate the rate per unit length by reference to related Ernst and C-metric instantons, concluding that it is completely negligible for GUT-scale strings. The estimated rate for macroscopic superstrings is much higher, although still extremely small, unless there is an early phase of strong coupling.
\end{abstract}

PACS numbers: 04.40.-b, 04.70.-s, 11.27+d

- Email: rg10012@amtp.cam.ac.uk

* Email: m.b.hindmarsh@sussex.ac.uk 
One of the basic tenets on which the cosmic string scenario [1-3] of galaxy formation rests is that the only way strings decay is via gravitational or particle radiation. The (straight) Nielsen-Olesen vortex [4] has been taken to be stable, protected by the topology of the vacuum manifold of the underlying Abelian Higgs model. Such topological arguments have been assumed to be true even when gravity is included. While NielsenOlesen vortices seem to be stable to small perturbations in Einstein Abelian Higgs [5], their stability against non-perturbative processes has not been confirmed.

Several recent papers have examined cosmic strings splitting under various conditions [6-8], but only [8] actually considered the splitting of a Nielsen-Olesen vortex. If true, these results might have far reaching consequences for any large scale application of cosmic strings if the decay rate is appreciable. All of these arguments use metrics with conical deficits to find an instanton for decay and assume the conical deficit can be smoothed out and replaced by a cosmic string. If a real vortex is to split it is crucial to show that a real vortex can be woven into these metrics, smoothly rounding off the conical deficit.

Evidence for the validity of replacing a conical deficit with a vortex was presented in [9], where it was shown that the metric of Aryal Ford and Vilenkin (AFV) [10], a black hole pierced by a conical deficit, could be considered as the thin string limit of a vortex piercing a black hole. The main difference between the AFV metric and the C-metrics, [11], used in [6-8] is that the latter represents a non-static process. From the technical point of view, the main difference is that in the one case (AFV) we have an almost cylindrically symmetric situation, whereas in the latter case, and also in the case of black holes in static equilibrium, there is an asymmetry of the system in that the string locally terminates on the event horizon. The string core no longer corresponds to " $r=0$ " in an axial coordinate system, but only " $r=0, z>z_{0}$ ". Thus, although the results of [9] were suggestive, they were by no means conclusive.

In this paper we demonstrate that the assumptions of [6-8] are justified, in the sense that we show how to replace the conical singularity of both the uncharged C-metric, as well as a static metric of a string with ends, with an abelian Higgs vortex. We will show that provided the mass of the black holes involved is sufficiently large, the NielsenOlesen solution can be used to approximate the field configuration, and the gravitational effect of the string will be shown to smooth out the conical singularity into a 'snub-nosed cone' [5]. A consequence of this is that Nielsen-Olesen vortices need not be stable to non-perturbative topology changing processes, and that strings might indeed 'split'.

We first consider the Nielsen-Olesen vortex and discuss the metrics on which it is supposed to sit. We then show how it smooths out any conical deficit by calculating the back reaction of the vortex on the geometry. After these more detailed considerations, 
we discuss some of the implications. One is that topologically stable strings can break as indicated in [8] by the nucleation of a pair of black holes, as the euclideanised version of the smoothed C-metric should represent an instanton by which a straight string can tunnel to the lorentzian configuration of black holes on Nielsen-Olesen vortices. The tunneling rate can be estimated to be proportional to $\exp \left(-\pi m^{2} / \mu\right)$, where $m$ is the mass of the black holes and $\mu$ the string tension. We point out that as far as strings at the Grand Unification scale are concerned, the tunneling rate is greatest for instantons where the black hole radius $2 m$ is much smaller than the thickness of the string $\sim \mu^{-1 / 2}$, and so the process of splitting is really described by a different metric, which should resemble the Ernst [12] metric in the vicinity of the black holes. We estimate the rate, and find it still to be negligibly small for Grand Unified scale strings, but of possible significance for cosmic superstrings [13], whose string tension is much higher. There is even the possibility of creating a population of primordial black holes, if the Universe ever went through a period where there was a significant population of macroscopic fundamental strings.

When Nielsen-Olesen strings were first conceived, they were meant to be a realisation of the Nambu action, which allows for both closed and open strings. The open strings would have to satisfy certain boundary conditions, namely that the ends travel at the speed of light. The reason that Nielsen-Olesen vortices were assumed not to have ends is associated with the topology of the vacuum manifold. For future reference, the abelian Higgs lagrangian is

$$
\mathcal{L}\left[\Phi, A_{\mu}\right]=D_{\mu} \Phi^{\dagger} D^{\mu} \Phi-\frac{1}{4} F_{\mu \nu} F^{\mu \nu}-\frac{\lambda}{4}\left(\Phi^{\dagger} \Phi-\eta^{2}\right)^{2}
$$

where $\Phi$ is a complex scalar field, $D_{\mu}=\nabla_{\mu}-i e A_{\mu}$ is the usual gauge covariant derivative, and $F_{\mu \nu}$ the field strength associated with $A_{\mu}$. We are using Planck units in which $G=$ $\hbar=c=1$ and a mostly minus signature. The vacuum manifold is $|\Phi|=\eta$, and therefore is a circle in the complex plane. The Nielsen-Olesen vortex takes the form

$$
\Phi=\eta X_{0}(r) e^{i \phi}, \quad ; \quad A_{\mu}=A_{0}(r) \nabla_{\mu} \phi
$$

in cylindrical polar coordinates. Now, note that

$$
\oint A_{\mu} d x^{\mu}=2 \pi A_{0}(r) \rightarrow \frac{2 \pi}{e} \quad \text { outside the core }
$$

where the line integral is taken to be at constant $r$. More generally, (i.e., if we are not in flat space, or do not have a straight string) the presence of a vortex is indicated by the existence of closed loops in space which lie totally in vacuo for which

$$
\oint A_{\mu} d x^{\mu}=\frac{2 \pi N}{e}
$$


for some $N \in \mathbb{Z}$. Then, Stokes' theorem is used to deduce the existence of a flux tube crossing any surface spanning the loop - and hence an infinite or closed string.

How then can a string have ends? One way, of course, is to embed the abelian Higgs model in a Yang-Mills-Higgs system with monopole solutions [1]. However, it was pointed out in [8] and [9] that as far as the topology of the fields is concerned, there is no obstruction to terminating the string on a black hole. The abelian gauge potential has to be defined in at least two patches on 2-spheres surrounding the black hole (just as in the Wu-Yang construction for magnetic monopoles [14]), so Stokes' theorem has to be used with care. The spacetime is not topologically trivial, (the particular measure here being here the second cohomology class), therefore we cannot conclude that flux crosses every surface spanning the loop - only those surfaces deformable to one known to contain a vortex. Thus, depending on the actual spatial topology, it is quite possible for a string to leave a neighbourhood and thus effectively terminate as far as a local observer is concerned.

Such a situation occurs in the other metrics considered by Aryal, Ford and Vilenkin, namely the C-metrics [11] and a modification of the static metrics considered by Israel and Khan [15]. A C-metric is an axially symmetric solution to the Einstein equations which represents two black holes uniformly accelerating apart. The force for this acceleration is provided either by a conical excess, a strut, between the holes, or alternatively by a conical deficit, a string, extending from each hole to infinity (or of course a combination of the two). The Israel-Khan metric represents two black holes held in equilibrium by a strut, but can be readily modified to have two strings extending to infinity. The key observation about these metrics is that the horizons of the two black holes can be identified, forming a wormhole in space $[11,16]$. The presence of this wormhole then provides a hole through which the string can exit, thus it is not necessary to consider charged black holes and topologically unstable strings, these uncharged metrics can directly 'swallow' a NielsenOlesen vortex. The basic idea then is to paint a vortex directly onto the metric, using the core to smooth out the conical deficit of the exact metric.

We will consider each metric in turn before showing that the Nielsen-Olesen vortex can smooth out the conical deficit. We will draw extensively on the formalism of [9] and refer the reader there for calculational details. There, the question of using the vortex to place hair on the black hole was considered, and it was shown that there was no obstruction to having a vortex sit on the event horizon. In fact, if the thickness of the vortex is less than the black hole radius $(E=\sqrt{\lambda} \eta m>1)$, the Nielsen-Olesen solution was shown to be an excellent approximation to the string fields and the vortex behaves almost as if the event horizon were not there. The fact that the event horizon appears to cut the string from an external observers point of view is readily explained by the open string boundary 
conditions. Recall that a Nambu string can end provided it is travelling at the speed of light. On the event horizon, the escape velocity is the speed of light, so a 'stationary' string sitting there is satisfying its appropriate boundary conditions. For $E<1$, numerical results showed that the string was still relatively unaffected by the black hole, although a slight pinching of the string does occur.

We now show that for small $\mu$, and string width much less than the black hole radius $(E \gg 1)$, the Nielsen-Olesen solution solves the abelian Higgs equations. We will neglect terms of order $\mu$ since these correspond to the back reaction of the geometry on the abelian Higgs equations, and can be accounted for via an iterative procedure.

First consider the C-metric which takes the form

$$
d s^{2}=A^{-2}(x+y)^{-2}\left[F(y) d t^{2}-F^{-1}(y) d y^{2}-G(x) d \phi^{2} / \kappa^{2}-G^{-1}(x) d x^{2}\right],
$$

where

$$
\begin{aligned}
& G(x)=1-x^{2}-2 m A x^{3}, \\
& F(y)=-1+y^{2}-2 m A y^{3} .
\end{aligned}
$$

Here, $m$ represents the mass of the black holes, and $A$ their acceleration. The factor $\kappa$ ensures that the axis between the black holes is regular, and $\phi$ has periodicity $2 \pi$. In the flat space limit, $A^{-1}$ represents half the distance of closest approach, so if this metric were to represent a string splitting, we would expect

$$
m \simeq \mu / A,
$$

where $\mu$ is the mass per unit length of the string. Let us write $x_{1}<x_{2}<x_{3}$ for the roots of $G$. Then, in order to obtain the correct signature, we must have $x_{2}<x<x_{3}$ and $-x_{2}<y<-x_{1}$. The coordinates cover only one patch of the full spacetime corresponding to the exterior spacetime of one accelerating hole up to its acceleration horizon, which is located at $y=-x_{2}$. The coordinate singularity at $y=-x_{1}$ corresponds to the event horizon of the black hole. The conical deficit sits along $x=x_{2}$, while $x=x_{3}$ points towards the other black hole, which means that $\kappa=\left|G^{\prime}\left(x_{3}\right)\right| / 2$. The magnitude of the deficit is given by

$$
\frac{\delta}{2 \pi}=1-\left|\frac{G^{\prime}\left(x_{2}\right)}{G^{\prime}\left(x_{3}\right)}\right|=\frac{x_{3}-x_{2}}{x_{3}-x_{1}} .
$$

Assuming $m A \ll 1$ in $(6)$, the three $\operatorname{roots}\left\{x_{i}\right\}$ are given by $\{-1 / 2 m A,-1,1\}$ so (8) requires $\delta=8 \pi m A$. But $\delta=8 \pi \mu$ for a string, hence $\mu=m A$ in agreement with (7). 
A more transparent form of the C-metric is obtained if we set

$$
\bar{t}=A^{-1} t, r=1 / A y, \text { and } \theta=\int_{x}^{x_{3}} d x / \sqrt{G}
$$

when

$$
d s^{2}=[1+\operatorname{Arx}(\theta)]^{-2}\left[\left(1-\frac{2 m}{r}-A^{2} r^{2}\right) d \vec{t}^{2}-\frac{d r^{2}}{\left(1-\frac{2 m}{r}-A^{2} r^{2}\right)}-r^{2} d \theta^{2}-r^{2} G d \phi^{2} / \kappa^{2}\right]
$$

This is almost conformally equivalent to the Kottler[18], or Schwarzschild de-Sitter metric as might be expected from the acceleration horizon and clearly shows that we reduce to the Schwarzschild metric in the limit $A \rightarrow 0$.

In order to solve the abelian Higgs equations, we rewrite the gauge field as

$$
A_{\mu}=\frac{1}{e}\left(\partial_{\mu} \phi-P_{\mu}\right)
$$

Using the expression (2) for $\Phi$, the equations of motion are then

$$
\begin{aligned}
\nabla_{\mu} \nabla^{\mu} X-P_{\mu} P^{\mu} X+\frac{\lambda \eta^{2}}{2} X\left(X^{2}-1\right) & =0 \\
\nabla_{\mu} F^{\mu \nu}+2 e^{2} \eta^{2} X^{2} P^{\nu} & =0 .
\end{aligned}
$$

where $F_{\mu \nu}=\partial_{\mu} P_{\nu}-\partial_{\nu} P_{\mu}$ now. Noting that in a normal spherically symmetric metric $X$ is a function of $r \sin \theta=\sqrt{g_{\phi \phi}}$, we try $X=X_{0}(R), P_{\phi}=P_{0}(R)$, where

$$
R=\frac{\sqrt{\lambda G} \eta r}{\kappa[1+A r x(\theta)]}
$$

in the neighbourhood of $x=x_{2}$, the cosmic string.

Note that

$$
r=\frac{1}{A y} \geq-\frac{1}{A x_{1}}=2 m
$$

and

$$
\frac{r}{1+A x r} \simeq \frac{1}{A\left(x_{2}+y\right)}>\frac{1}{A\left(x_{2}-x_{1}\right)}=\frac{2 m}{1-2 \mu}
$$

hence

$$
\frac{\sqrt{\lambda} \eta r}{[1+\operatorname{Ar} x(\theta)]}>2 E \gg 1
$$


We therefore are interested in examining the equations of motion over a range

$$
\frac{\sqrt{G}}{\kappa} \leq 1 / 2 E \ll 1
$$

Thus

$$
\begin{aligned}
\frac{\partial X}{\partial r} & =\frac{\sqrt{\lambda G} \eta}{\kappa[1+A x r]^{2}} X^{\prime}(R)=\sqrt{\lambda} \eta X^{\prime}(R) \times O\left(E^{-1}\right) \\
\text { and } \quad \sqrt{G} \frac{\partial X}{\partial \theta} & =\frac{\sqrt{\lambda G} \eta r}{\kappa[1+A x r]} X^{\prime}(R)\left[\frac{G^{\prime}(x)}{2}-\frac{A r G}{(1+A x r)}\right]=R X^{\prime}(R)\left[1+O\left(\mu E^{-2}\right)\right]
\end{aligned}
$$

Putting this form of $X$ into the $\mathrm{X}$-equation of motion yields

$$
X^{\prime \prime}\left[1+O(\mu)+O\left(E^{-2}\right)\right]+\frac{X^{\prime}}{R}\left[1+O(\mu)+O\left(E^{-2}\right)\right]+\frac{X P^{2}}{R^{2}}+\frac{1}{2} X\left(X^{2}-1\right)=0,
$$

which is indeed the Nielsen-Olesen equation for $X$ to the required order. The equation for $P$ works similarly. Hence the Nielsen-Olesen vortex can be "painted" on to the C-metric, in spite of its non-static nature.

Now consider the Israel-Khan metric which is given by

$$
d s^{2}=e^{2 \psi_{o}} d t^{2}-e^{2\left(\gamma_{o}-\psi_{o}\right)}\left(d r^{2}+d z^{2}\right)-r^{2} e^{-2 \psi_{o}} d \phi^{2},
$$

where, writing

$$
\begin{aligned}
\zeta & =\sqrt{\lambda} \eta z \quad ; \quad \rho=\sqrt{\lambda} \eta r \\
\zeta_{1} & =\zeta-\left(L+\frac{1}{2} E\right) \quad ; \quad \zeta_{1}^{\prime}=\zeta-\left(L-\frac{1}{2} E\right) \\
\zeta_{2} & =\zeta+\left(L-\frac{1}{2} E\right) \quad ; \quad \zeta_{2}^{\prime}=\zeta+\left(L+\frac{1}{2} E\right) \\
R_{1}^{2} & =\left(\rho^{2}+\zeta_{1}^{2}\right) \quad \text { etc. } \\
E(i, j) & =R_{i} R_{j}+\left(\zeta_{i} \zeta_{j}+\rho^{2}\right) \quad ; \quad E\left(i^{\prime}, j\right)=R_{i} R_{j}+\left(\zeta_{i}^{\prime} \zeta_{j}+\rho^{2}\right)
\end{aligned}
$$

we have

$$
\psi_{o}=\frac{1}{2} \log \left[\frac{R_{1}+R_{1}^{\prime}-E}{R_{1}+R_{1}^{\prime}+E}\right]\left[\frac{R_{2}+R_{2}^{\prime}-E}{R_{2}+R_{2}^{\prime}+E}\right]
$$

and

$$
\gamma_{o}=\frac{1}{4} \log \left[\frac{E\left(1^{\prime}, 1\right)^{2} E\left(1^{\prime}, 2\right)^{2} E\left(1,2^{\prime}\right)^{2} E\left(2,2^{\prime}\right)^{2}}{E(1,1) E\left(1^{\prime}, 1^{\prime}\right) E(1,2)^{2} E\left(1^{\prime}, 2\right)^{2} E(2,2) E\left(2^{\prime}, 2^{\prime}\right)}\right]-\log \frac{4 L^{2}-E^{2}}{4 L^{2}}
$$


where $E=\sqrt{\lambda} \eta m \gg 1$ represents (half) the black hole radius in multiples of string width and $L$ represents the separation of the black holes, also in units of string width. We can directly find $L$ given the energy per unit length of the string, since the conical deficit for $\zeta>L+\frac{1}{2} E$ is

$$
\begin{aligned}
4 \mu & =\frac{\delta}{2 \pi}=1-e^{-\gamma_{o}(\rho=0)}=\frac{E^{2}}{4 L^{2}} \\
& \Rightarrow L=\frac{E}{4 \sqrt{\mu}} \gg 1
\end{aligned}
$$

Now consider the string extending from the upper black hole to infinity. We are then interested in a coordinate range $\rho<\rho e^{-\psi_{o}} \leq O(1)$ and $\zeta>L+\frac{1}{2} E$. Thus

$$
R_{2}+R_{2}^{\prime}=2(\zeta+L)+O(\mu)+O\left(E^{-2}\right)
$$

hence

$$
\frac{R_{2}+R_{2}^{\prime}-E}{R_{2}+R_{2}^{\prime}+E}=1-\frac{E}{\zeta+L}+O(\mu)
$$

Denote this quantity by $\log \psi_{2}$. Then $\psi_{2}=O(\sqrt{\mu})$ and $\psi_{2, z}=\sqrt{\lambda} \eta \times O(\mu / E)$. Similarly, $\gamma=\gamma_{\text {sch }}+\gamma_{2}$ where $\gamma_{2}$ is $\mathrm{O}(\sqrt{\mu})$ and has a similarly suppressed variation. Thus the effect of the second black hole is to multiply the Schwarzschild metric in the vicinity of the string core by an extremely slowly varying factor. Therefore within the stated limits of the approximation $(E \gg 1)$ the metric is Schwarzschild up to non relevant factors, and the results of [9] can be used to conclude that the vortex equations can be solved to the required order by the Nielsen-Olesen solution.

Now we turn to the gravitational back reaction. For this we use the canonical form of a general axisymmetric metric [19]

$$
d s^{2}=e^{2 \psi} d t^{2}-e^{2(\gamma-\psi)}\left(d z^{2}+d r^{2}\right)-\tilde{\alpha}^{2} e^{-2 \psi} d \phi^{2}
$$

Note that although the Israel-Khan metric (20) is already in this form, the C-metric is not. In order to make it so, one must perform the coordinate transformation

$$
r=\frac{\sqrt{F G}}{\kappa A^{2}(x+y)^{2}} \quad ; \quad z=-\frac{\left(1+x y+m A\left(x^{3}+2 x^{2} y-y^{3}-2 x y^{2}\right)\right)}{\kappa A^{2}(x+y)^{2}}
$$

In which case the metric comes into the canonical form of equation (20) with

$$
\begin{aligned}
e^{2 \psi_{o}} & =\frac{F}{A^{2}(x+y)^{2}} \\
e^{-2 \gamma_{o}} & =\frac{r^{2} A^{4}(x+y)^{4}}{F}\left[F\left(\frac{F^{\prime}(y)}{2 F}-\frac{2}{(x+y)}\right)^{2}+G\left(\frac{G^{\prime}(x)}{2 G}-\frac{2}{(x+y)}\right)^{2}\right]
\end{aligned}
$$


Although this appears rather messy, the key facts are that it does have the canonical form, and that

$$
e^{-2 \gamma_{o}(r=0)}=\frac{G^{\prime}\left(x_{2}\right)^{2}}{4 \kappa^{2}}=\frac{G^{\prime}\left(x_{2}\right)^{2}}{G^{\prime}\left(x_{1}\right)^{2}} .
$$

Now, writing

$$
\begin{aligned}
\rho & =\sqrt{\lambda} \eta r, \\
\zeta & =\sqrt{\lambda} \eta z, \\
\alpha & =\sqrt{\lambda} \eta \tilde{\alpha} .
\end{aligned}
$$

the relevant Einstein equations from [9] are

$$
\begin{aligned}
\alpha_{, \zeta \zeta}+\alpha_{, \rho \rho} & =-\epsilon \sqrt{-g}\left(\hat{T}_{\zeta}^{\zeta}+\hat{T}_{\rho}^{\rho}\right) \\
\left(\alpha \psi_{, \zeta}\right)_{, \zeta}+\left(\alpha \psi_{, \rho}\right)_{, \rho} & =\frac{1}{2} \epsilon \sqrt{-g}\left(\hat{T}_{0}^{0}-\hat{T}_{\zeta}^{\zeta}-\hat{T}_{\rho}^{\rho}-\hat{T}_{\phi}^{\phi}\right) \\
\gamma_{, \rho \rho}+\gamma_{, \zeta \zeta} & =-\psi_{, \rho}^{2}-\psi_{, \zeta}^{2}-\epsilon e^{2(\gamma-\psi)} \hat{T}_{\phi}^{\phi}
\end{aligned}
$$

where $\epsilon=8 \pi \eta^{2}$ represents the gravitational strength of the string, and $\hat{T}_{b}^{a}=T_{b}^{a} / \lambda \eta^{4}$ is a normalised energy momentum tensor which is of order unity. The combinations appearing above were all shown to be functions of $R=\rho e^{-\psi_{o}}$ in [9]. The slight alteration of the metric will not change this. The main difference between the current calculation and the one presented in [9] is of course the asymmetry. In [9] the Einstein equations were integrated out from $r=0$ to obtain an asymptotically conical metric. Here we do not expect a conical deficit over the full range of $\zeta$, therefore it is more appropriate to place boundary conditions at the edge of the string rather than at its core. That is, we take the metric perturbation to be non-zero only in the core.

Writing the metric functions as

$$
\gamma=\gamma_{0}+\epsilon \gamma_{1} \quad \psi=\psi_{0}+\epsilon \psi_{1} \quad \alpha=\rho\left(1+\epsilon \alpha_{1}\right)
$$

we expect $\alpha_{1}, \gamma_{1}, \psi_{1} \rightarrow 0$ outside the core. Solving the Einstein equations then gives

$$
\begin{aligned}
& \alpha_{1}(R)=-\int_{R}^{\infty} \frac{1}{R^{2}} \int_{0}^{R} R^{2}\left[\hat{T}_{0}^{0}+\hat{T}_{\rho}^{\rho}\right] d R \\
& \psi_{1}(R)=-\frac{1}{2} \int_{R}^{\infty} R \hat{T}_{\rho}^{\rho}=\frac{1}{2} \gamma_{1}(R)
\end{aligned}
$$


over the relevant ranges of $\zeta$. Note that the perturbations fall to zero outside the core*, leaving the background metric (Israel-Khan or $\mathrm{C}$ ), but what of the interior metric? The real issue is what happens as $\rho \rightarrow 0$. Here there will be a nodal singularity if

$$
\delta=2 \pi\left(1-\alpha^{\prime}(0) e^{-\gamma(0)}\right)=2 \pi\left(1-\left(1+\epsilon \alpha_{1}(0)\right)\left(1-\epsilon \gamma_{1}(0)\right) e^{-\gamma_{o}(0)}\right) \neq 0
$$

But

$$
\alpha_{1}(0)-\gamma_{1}(0)=-\int_{0}^{\infty} R \hat{T}_{0}^{0} d R=4 \mu / \epsilon
$$

and in each case, the background metric was chosen to have a nodal singularity (see equations (24) and (8)) of $8 \pi \mu$ along this axis, i.e.,

$$
1-e^{-\gamma_{o}(0)}=4 \mu
$$

hence $\delta=0$ to order $\mu$. Thus the effect of the vortex is to smooth out the conical singularity giving a regular metric with a snub-nosed cone which is schematically depicted in figure 1.

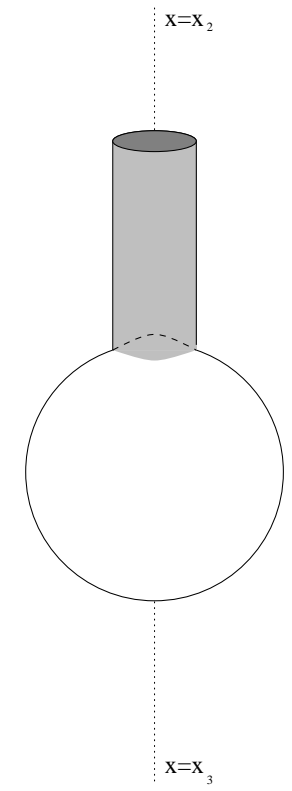

FIGURE (1): Pictorial representation of the geometry of the vortex terminating on the black hole.

* Note however that $\alpha_{1}$ actually falls to zero as $O\left(E^{-1}\right) / R[9]$. Although this is outside the scope of our present approximation, it is tempting to speculate that some relic of this might remain when the calculation is continued to higher orders, and perhaps perturb the gravitational radiation present in the C-metrics [11], rather similar to the way in which a string in a FRW universe radiates C-energy as it preserves its proper radius [20]. 
We have therefore shown that the Nielsen-Olesen solution can be used to construct regular metrics (in the sense of no nodal singularities) which represent vortices which end on black holes either in static equilibrium, or accelerating off to infinity. This latter metric gives the appearance of a cosmic string being eaten up by accelerating black holes. Whether or not such a process can be used to destabilize cosmic strings depends on the action of the corresponding instanton obtained by euclideanising the C-metric. Note that in a model with vortices, we no longer require equality of the periodicities of euclidean time at the event and acceleration horizon [17], since we can always dress one or other horizon with an appropriate virtual string worldsheet which can eat up any excess in periodicity of imaginary time [21], just as the lorentzian string 'eats up' the $\phi$-angle. Indeed, since we are considering Einstein-abelian-Higgs a priori, constructing such Euclidean vortices[21] to consume $\tau$-intervals in the black holes geometry is a natural procedure to undertake. It is arguably preferable to leaving a conical deficit there.

Euclideanizing (5) gives natural periodicities at the event and acceleration horizons of

$$
\beta_{e}=\frac{4 \pi}{\left|G^{\prime}\left(x_{1}\right)\right|} \text { and } \quad \beta_{a}=\frac{4 \pi}{\left|G^{\prime}\left(x_{2}\right)\right|}
$$

Combining this and equation (8) we can immediately see that demanding equality of these periodicities requires $\mu=1 / 4$. Thus for small $\mu, \beta_{e} \neq \beta_{a}$. In fact

$$
\beta_{e} \simeq 8 \pi \mu \gg \beta_{a} \simeq 2 \pi
$$

So it appears that it is the acceleration horizon that must be dressed if we wished to have a completely regular Euclidean section. This would appear to imply that the action for such a process is infinite, however, since calculations of the euclidean action are delicate [22], it would be premature to conclude that this must be the case.

With this caveat in mind, let us assume that the action is finite, and estimate it. The instanton has the appearance of a two-dimensional plane (the string worldsheet) with a disc, of radius $\rho$ say, removed from it. The difference between the action of such a configuration and that of the planar string worldsheet is roughly

$$
I(\rho)=2 \pi \rho m-\pi \mu \rho^{2} .
$$

Extremizing this action with respect to $\rho$ gives the critical radius $\rho_{\mathrm{c}}=m / \mu$, and therefore the value of the action at the critical point is

$$
I_{\mathrm{c}}=\pi m^{2} / \mu \text {. }
$$


However, in order to approximate the C-metric we had to assume that the vortex was much thinner than the width of the hole, or

$$
m>\frac{1}{\sqrt{\lambda} \eta} \sim \mu^{-1 / 2} .
$$

Thus, for Grand Unified strings, we are perforce considering instantons with enormous values of $I_{\mathrm{c}}$ : in fact,

$$
I_{\mathrm{c}} \geq O\left(\mu^{-2}\right) \sim 10^{12}
$$

in agreement with the estimates of [8].

In order to reduce the action below this value we have to reduce the size of the black hole below that of the string, and thus move away from the C-metric. Recall that, apart from some pinching, the vortex is essentially unaffected by the black hole horizon. This means that in the limit $m \ll 1 / \sqrt{\lambda} \eta$, the black holes can be contained entirely within the vortex, where the magnetic field is fairly uniform. In fact, near the centre of the vortex, the strength of the magnetic field is $B=B_{\mathrm{s}} \simeq 2 \pi / e m_{\mathrm{v}}^{2}$, where $m_{\mathrm{v}}=\sqrt{2} e \eta$ is the mass of the gauge field. We would expect this magnetic field to be able to nucleate magnetically charged black holes, and that the instanton describing this process to resemble locally the euclideanised Ernst metric [16,17] rather than the C-metric. The Ernst metric [12], we recall, is an exact solution to the coupled Einstein-Maxwell field equations, describing a pair of oppositely charged black holes accelerating under the influence of a magnetic field. However, the real metric describing a string splitting into a pair of small black holes in the Einstein-Abelian-Higgs system must confine the magnetic flux to within a distance $m_{\mathrm{v}}^{-1}$ of the axis of symmetry, and thus we would expect to recover the C-metric at spacelike separations much greater than this. At intermediate scales, the flux lines emanating from the black hole will get swept up and around into the confined vortex extending to infinity, as in figure 2.

The extra action for the creation, separation, and annihilation of a pair of virtual black holes in the background field $B$ can be estimated as

$$
I(\rho)=2 \pi m \rho-\pi q B \rho^{2},
$$

where $q=2 \pi / e$ is the magnetic charge of the black hole. This is extremised at $\rho_{\mathrm{c}}=m / q B$, so

$$
I_{\mathrm{c}}=\pi m^{2} / q B
$$

In the background supplied by the core of the vortex, we find (using $\mu=2 \pi \eta^{2}$, which is only strictly true in when $\lambda=e^{2} / 2$ )

$$
I_{\mathrm{c}}=m^{2} / 2 \mu \text {. }
$$




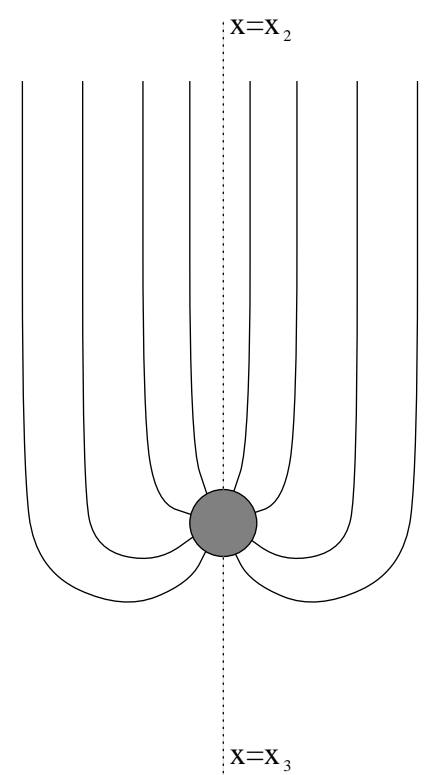

FIGURE (2): A representation of the nucleation of a small black hole within

a thick vortex.

This is different by a factor of $2 \pi$ from the C-metric action extrapolated beyond its domain of validity [6]. The minimum possible action is obtained when the hole is extremal, $m=q$, for which

$$
I_{\mathrm{c}}=2 \pi / e^{2} \mu,
$$

which for Grand Unified strings is of order $10^{7}$.

With this action we can estimate the rate per unit length of string $\gamma$ for the breaking process as

$$
\gamma \sim M^{2} \exp \left(-2 \pi / e^{2} \mu\right)
$$

where $M$ is a mass scale in the problem. This can only be calculated by evaluating the determinants of small fluctuations in the instanton background, which is beyond the scope of the present paper. However, we can estimate the prefactor by drawing on the known rate per unit volume for particle creation in a uniform electric field $E$ [23], which is $\left(e^{2} E^{2} / 8 \pi^{3}\right) \exp \left(-\pi m^{2} / e E\right)$. Since the field is confined to a tube of area $m_{\mathrm{v}}^{-2}$, the rate should be approximately

$$
\gamma \sim e^{-4} \mu \exp \left(-2 \pi / e^{2} \mu\right)
$$

This rate is utterly negligible for GUT-scale strings. Even if the Universe was crammed with strings, so that they begin to overlap, the rate of pair creation would still be only of order $\mu^{2} t^{3} \exp \left(-2 \pi / e^{2} \mu\right) \sim 10^{200} \exp \left(-10^{6}\right)$. 
The best we can hope to do is to put a macroscopic superstring at the conical deficit of the C-metric. Of course, we should in that case solve the low-energy superstring field equations, including the dilaton and the antisymmetric tensor field. (This would be very similar to the calculation of [17], however with an axion, rather than electromagnetic, field.) However, it is known that the metric around a superstring is also conical [24], so it is not at all inconceivable that an exact solution (and an associated instanton) similar to the C-metric exists in this theory.

The mass per unit length of a macroscopic superstring is $g^{2} / 32 \pi^{2}$ [25], where $g$ is the Grand Unified gauge coupling, which is of order $10^{-3}$ at the GUT scale. We could not reasonably expect the black holes to have less than the Planck mass, and so the exponential factor is of order $\exp \left(-10^{3}\right)$. The entire history of the visible universe occupies only about $10^{240}$ Planck units of spacetime volume, and so the splitting process is negligible even for superstrings, unless there is a period where the gauge coupling constant is large. In that case, there might be a relic population of primordial black holes left behind by an early phase of breaking superstrings.

To summarise: we have demonstrated that it is possible to replace the conical singularities of the C-metrics and the Israel-Khan metric with a vortex solution of the abelian Higgs model by calculating the gravitational back reaction to linear order in $\mu$, the energy per unit length of the string. We consider the implications for the splitting of cosmic strings and argue that if the decay does proceed by an instanton in the euclidean theory, then it will in any case be suppressed by a ludicrously large factor even for GUT strings. The tunnelling process for cosmic superstrings, which have a yet larger string tension, is still extremely small. This suggests that although it is of great interest that otherwise topologically stable strings might be unstable, it is probably of no relevance to practical applications.

Note added in proof. We thank G.Horowitz for pointing out a problem with our suggestion of splitting superstrings, namely, that the axion field has a conserved topological charge. We have left this argument in case the reader can find a resolution. We would also like to thank Simon Ross for pointing out an error in the initial version of the manuscript.

\section{Acknowledgements}

We would like to thank Ana Achucarro and Konrad Kuijken for conversations. This work is supported by PPARC Advanced Research Fellowships B/93/AF/1716 (R.G.) and B/93/AF/1642 (M.H.).

\section{References}

[1] M.B. Hindmarsh and T.W.B. Kibble, Rep. Prog. Phys. 58, 477 (1995). 
[2] A. Vilenkin and E.P.S. Shellard, Cosmic strings and other Topological Defects (Cambridge Univ. Press, Cambridge, 1994).

[3] R.H. Brandenberger, Modern Cosmology and Structure Formation astro-ph/9411049.

[4] H.B. Nielsen and P. Olesen, Nucl. Phys. B61, 45 (1973).

[5] R. Gregory, Phys. Rev. Lett. 59, 740 (1987).

[6] S.W. Hawking and S.F. Ross, Pair production of black holes on cosmic strings, grqc/9506020.

[7] R. Emparan, Pair creation of black holes joined by cosmic strings, gr-qc/9506025.

[8] D. Eardley, G. Horowitz, D. Kastor and J. Traschen, Breaking cosmic strings without monopoles, gr-qc/9506041.

[9] A. Achucarro, R. Gregory and K. Kuijken, Abelian Higgs Hair for Black Holes, grqc/9505039.

[10] M. Aryal, L. Ford and A. Vilenkin, Phys. Rev. D34, 2263 (1986).

[11] W. Kinnersley and M. Walker, Phys. Rev. D2, 1359 (1970).

[12] F.J. Ernst, J. Math. Phys. 17, 515 (1976).

[13] E. Witten, Phys. Lett. B153, 243 (1985).

[14] T.T. Wu and C.N. Yang Phys. Rev. D12, 3845 (1975, Nucl. Phys. B107, 365 (1976)).

[15] W. Israel and K.A. Khan, Nuovo Cim. 33331 (1964).

[16] D. Garfinkle and A. Strominger, Phys. Lett. B256, 146 (1991).

[17] H.F. Dowker, J.P. Gauntlett, D.A. Kastor and J. Traschen, Phys. Rev. D49, 2909 (1994).

[18] F.Kottler, Ann. Phys. (Leipzig) 56, 401 (1918).

[19] J.L. Synge, Relativity: The General Theory (North Holland, Amsterdam, 1960).

[20] R.Gregory, Phys. Rev. D39, 2108 (1989).

[21] H.F. Dowker, R. Gregory and J. Traschen, Phys. Rev. D45, 2762 (1992).

[22] S.W.Hawking and G.T.Horowitz, The Gravitational Hamiltonian, action, entropy and surface terms, gr-qc/9501014.

[23] C. Itzykson and J-B. Zuber, Quantum Field Theory (McGraw-Hill, New York, 1980).

[24] A. Dabholkar, G. Gibbons, J.A. Harvey and F. Ruiz Ruiz, Nucl. Phys. B340, 33 (1990).

[25] N. Turok, Phys. Rev. Lett. 60, 549 (1988). 